\title{
Primary prevention of cardiovascular disease: an umbrella review of non-pharmacological interventions
}

\section{Prevención primaria de enfermedades cardiovasculares: una revisión de revisiones de intervenciones no farmacológicas}

doi.org/10.23938/ASSN.0316

\author{
E. Martín-Ruiz ${ }^{1}$, A. Olry-de-Labry-Lima ${ }^{1,2,3}$, D. Epstein ${ }^{4}$
}

\begin{abstract}
Background. The aim of this study is to determine the effectiveness of non-pharmacological interventions for prevention of cardiovascular disease (CVD) events and mortality in healthy adults or those at high risk of CVD.

Methods. An umbrella review about primary prevention of non-pharmacological interventions was undertaken in key databases as PubMed Health, Effective Health Care Program AHRQ, McMaster University and the Cochrane Plus until July 2017. The primary outcomes were the relative risk of fatal and non-fatal CVD events, and mortality. Secondary outcomes were adverse events.

Results. Twenty-four reviews were included of which thirteen reported outcomes of interest. Four of these found a pooled statistically significant risk reduction: dietary supplements of vitamin D, increased consumption of omega 3 fatty acids, Qigong, and counselling or education to modify more than one cardiovascular risk factor. Seven studies reported adverse events but minor or insignificant with respect to the control group.

Conclusions. Four non-pharmacological interventions have been shown to provide a statistically significant reduction in risk of CVD events or overall mortality, with minor adverse events if any. Further research should aim for higher methodological quality and longer follow-up of interventions to establish if these interventions, alone or in combination, translate into definite long-term health benefits.
\end{abstract}

Keywords. Cardiovascular. Non-prescription intervention. Umbrella review. Systematic reviews and meta-analyses.

\section{RESUMEN}

Fundamento. El objetivo de este estudio es determinar la efectividad de intervenciones no farmacológicas para la prevención de eventos relacionados con enfermedad cardiovascular (CVD), y mortalidad en adultos sanos o con alto riesgo de CVD.

Método. Revisión de revisiones sobre prevención primaria de intervenciones no farmacológicas en las bases de datos de PubMed Health, Effective Health Care Program AHRQ, McMaster University and the Cochrane Plus hasta Julio de 2017. Los resultados primarios fueron el riesgo relativo de eventos CVD, fatales y no fatales, y de mortalidad; los efectos adversos fueron los eventos secundarios.

Resultados. Se incluyeron veinticuatro revisiones, de las cuales trece informan resultados de interés. Cuatro observaron una reducción significativa del riesgo: suplementación de vitamina $\mathrm{D}$, incremento del consumo de ácidos grasos omega 3, Qigong, y educación para la modificación de factores de riesgo cardiovascular. Siete revisiones informaron sobre eventos adversos menores y/o no significativos respecto del grupo control.

Conclusiones. Cuatro intervenciones no farmacológicas son las que muestran una reducción estadísticamente significativa en eventos CVD o mortalidad total, con escasos o nulos efectos adversos. Se requiere de una investigación de mayor calidad metodológica y de mayor seguimiento de las intervenciones para establecer si estas, combinadas o por separado, conducen a claros beneficios en salud a largo plazo.

Palabras clave. Cardiovascular. Intervención no-prescrita. Revisión de revisiones. Revisiones sistemáticas y meta-análisis.
1. Escuela Andaluza de Salud Pública (EASP). Granada.

2. CIBER en Epidemiología y Salud Pública (CIBERESP).

3. Instituto de Investigación Biosanitaria. Granada. Hospitales Universitarios de Granada. Universidad de Granada.

4. Facultad de Ciencias Económicas. Universidad de Granada.
Recepción: 11/12/2017

Aceptación provisional: 19/04/2018

Aceptación definitiva: 03/08/2018

\author{
Correspondencia: \\ Antonio Olry de Labry \\ Escuela Andaluza de Salud Pública \\ Campus Universitario de Cartuja \\ Cuesta del Observatorio, 4 \\ 18080 Granada \\ E-mail: antonio.orlylabry.easp@juntadeandalucia.es
}

\section{Financial support}

This study was financed by the EPIC-CVD project under the European Commission. Directorate General for Research \& Innovation FP 7 Health 2011. Grant Agreement number 279233.

Systematic review registration: Prospero CRD 42015016447 


\section{INTRODUCTION}

Statins and other pharmacological interventions are now widely recommended as primary prevention against cardiovascular disease $(\mathrm{CVD})^{1}$. Yet while statins were initially recommended for high-risk individuals, this threshold has steadily been reduced. For example, guidelines from the National Institute for Care Excellence imply that ten million healthy individuals in the UK, with no previous history of CV events, are now candidates for taking statins for primary prevention $^{2}$. This has led to concerns about potential long term risks and over-medicalization ${ }^{3}$.

Consequently, attention is shifting to non-pharmacological interventions for primary prevention, which many perceive as more appropriate for healthy individuals, with less risk of harm, and possibly with lower overall cost than statins to the public health system. Undoubtedly, tobacco cessation, reducing dietary fats and increasing physical activities have played a part in reducing rates of $\mathrm{CVD}^{4-5}$. According to the WHO, appropriate policy and environmental changes could bring about further major reductions. Nevertheless, there is less evidence about which non-pharmacological interventions led by primary care professionals have been shown effective for CVD risk reduction in the general population as an alternative (or complement) to widespread use of statins.

Many candidate non-pharmacological interventions have been studied, including dietary advice, food supplements with vitamins or minerals, physical exercise, mental exercises and general health checks. The objective of this study is to determine the effectiveness of non-pharmacological interventions for prevention of cardiovascular disease and mortality in people with no history of CVD events. We apply an umbrella review methodology. This is a "review-of-reviews", providing a balanced summary of evidence from several systematic reviews of randomised controlled trials. This enables us to take a broad view of the state of the evidence at a given point in time across a wide range of different treatments. The study is carried out according to methodological recommendations from the Joanna Briggs Institute ${ }^{6}$ and Smith et $\mathrm{al}^{7}$.

\section{MATERIALS AND METHODS}

The research question was to determine the relative effectiveness of non-pharmacological interventions to prevent major CVD events and mortality in adult classified as having no previous history of CVD events or at risk of developing CVD, compared to placebo or no intervention.

The design of the study, an umbrella review of the literature, was carried out. The protocol was registered in PROSPERO (CRD 42015016447 http://www.crd.york.ac.uk).

The search was conducted using $\mathrm{Pu}-$ bMed Health, Effective Health Care Program Agency for Healthcare Research and Quality (AHRQ), Health Evidence and the Cochrane Plus databases; and using as free terms: "primary prevention", "cardiovascular disease", "review". In addition, the reference lists of the included articles were examined. Only papers in English or Spanish and published by 30th July 2017 were included.

Inclusion criteria:

- systematic reviews of randomized clinical trials (with or without meta-analysis)

- adult classified as having no history of CVD events, or at risk of developing CVD events or those of general population

- intervention: Non-pharmacological for targeted interventions to improve cardiovascular health in primary prevention. Any duration or time of follow- up periods were considered

- comparing group: placebo or no intervention

- outcomes intended to be measured: overall mortality, cardiovascular mortality, cardiovascular events, and adverse events related with the interventions

Exclusion criteria:

- systematic reviews conducted only in populations with specific conditions (only smokers, only obese people)

- reviews which mix primary and secondary prevention populations together 
- review which mix results of clinical trials with other designs

- systematic reviews of quasi-experimental designs

Identification of the reviews was carried out by two reviewers (EMR and AOL) who selected independently all the works and checked both title and abstract against the inclusion and exclusion criteria. Any discrepancies were agreed by consensus or third researcher (DE). Any duplicate articles were subsequently excluded. Articles were managed using Reference Manager Software.

Following the definitive selection of the articles of interest, two researchers carried out an independent evaluation of the methodological quality of each review (EM and AOL). To achieve this, the Assessing the Methodological Quality of Systematic Reviews (AMSTAR) checklist with eleven items was used ${ }^{8}$. Any discrepancies were discussed and agreed by consensus.

A data extraction form was developed to record bibliographic information. The general characteristics of the study (participants included, description of intervention) and outcome data (including details of outcome assessment and adverse effects) were extracted from each included study. In those articles that reported data for both primary and secondary prevention, we extracted only the primary prevention results.

The authors carried out a qualitative synthesis of results from included reviews, the interventions were classified according to focus of intervention (proposed by the authors), and type of outcomes (all-cause mortality, cardiovascular mortality, both fatal or non-fatal CVD events and adverse events). Heterogeneity between studies was evaluated using Higgin's $\mathrm{I}^{2}$, where $\mathrm{I}^{2}>50 \%$ imply a substantial level of heterogeneity 9 . A random-effects model was used to combine study-specific relative risk (RR) and calculate a 95\% confidence interval (CI). The analyses were performed with Stata software version 11.

\section{RESULTS}

The initial search yielded a total of 643 publications. After excluding by title and/or abstract, a total of 127 full-text works were assessed for eligibility (see Figure 1). One review ${ }^{10}$ was excluded in order to avoid duplicity, as it had the same aim and higher risk of bias than another included review ${ }^{11}$. Finally, twenty-four reviews were included in the final synthesis (Fig. 1), of which thirteen reported CVD events and/or overall mortality as primary outcome.

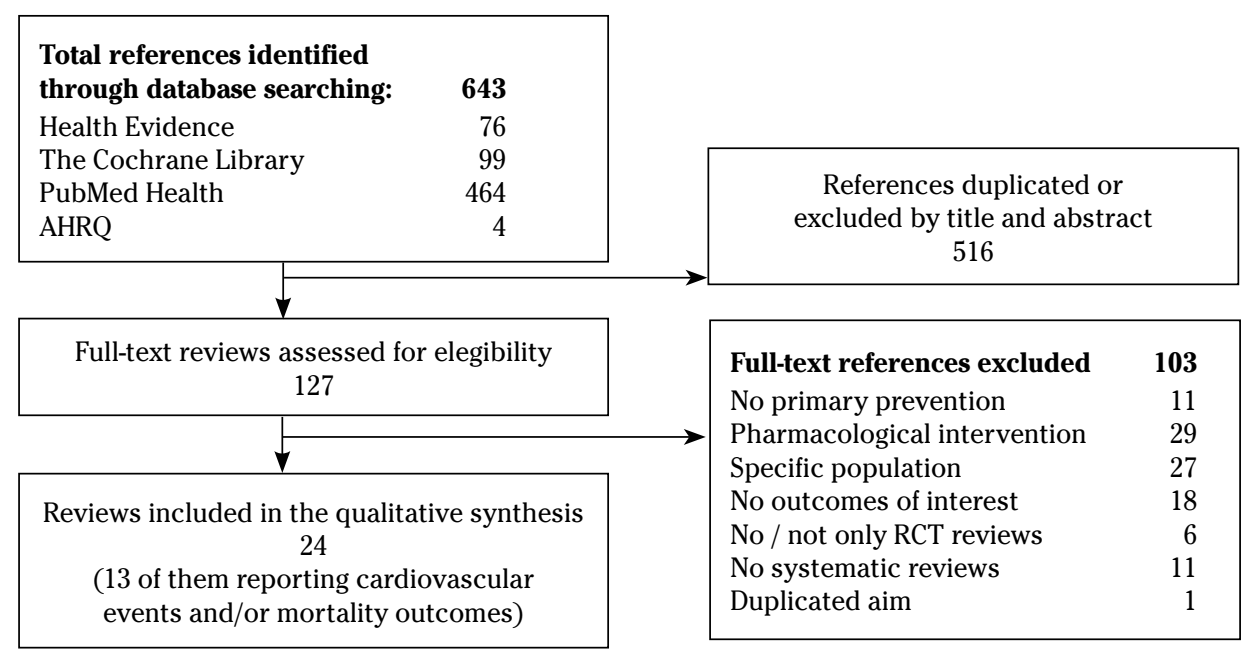

Figure 1. Flow diagram for review. 


\section{Primary prevention}

Eight types of non-pharmacological interventions for primary prevention of CVD were reviewed (Table 1):

- supplements: vitamin $\mathrm{C}^{12}$, antioxidant $^{13}$, vitamin $\mathrm{D}^{14}$, selenium ${ }^{15}$, vitamin Co-enzyme $\mathrm{Q} 10^{16}$, vitamin $\mathrm{K}^{17}$, and vitamin, mineral and multivitamin supplements $^{18}$. Fortmann's review ${ }^{22}$ included different interventions based on single and paired combination of supplementation with vitamins and minerals supplementation, including vitamin $\mathrm{C}$ and selenium. The two reviews on vitamin $\mathrm{C}^{12,18}$ shared elements such as type of population included and intervention, but they differed in both the outcome measures included and some inclusion criteria (minimum follow-up time for required randomized controlled trials (RCT) (three months) ${ }^{12}$, trials with good or fair quality, studies carried out in countries whose Human Development Index was considered very high $^{18}$ )
- dietary interventions: advice to reduce dietary salt ${ }^{19}$, advice to increased consumption of fruit and vegetables $^{20}$ and Mediterranean dietary pattern ${ }^{21}$

- diet supplementation: nuts, green and black tea ${ }^{23}$, dietary fibre ${ }^{24}$, Omega $3^{25}$ and Omega $6^{26}$ fatty acids

- multiple risk factor interventions: counseling or education to modify more than one cardiovascular risk factor $^{11,27}$, the last of them in low income countries

- physical exercise: exercise training ${ }^{28}$, Qigong $^{29}$, Tai chi $^{30}$, Yoga ${ }^{31}$

- mental exercise: transcendental meditation $^{32}$

- digital health intervention ${ }^{33}$

- general health checks ${ }^{34}$

Twenty-two reviews were published by the Cochrane collaboration and two by other groups ${ }^{18,33}$, all during the last five years, except two reviews from $2004^{20,25}$ were not published in the last five years (Table 1). The overall number of participants included in these systematic reviews ranged between 60 participants

Table 1. Characteristics of the reviews included and adverse events

\begin{tabular}{|c|c|c|c|c|c|c|c|}
\hline $\begin{array}{l}\text { Author } \\
\text { Year }\end{array}$ & $\begin{array}{l}\text { Intervention } \\
\text { Comparison }\end{array}$ & Population & Outcomes & $\begin{array}{c}\text { Studies } \\
\text { Participants }\end{array}$ & $\begin{array}{l}\text { Follow-up } \\
\text { Range }\end{array}$ & $\begin{array}{l}\text { Characteristics } \\
\text { of Participants }\end{array}$ & $\mathrm{AE}$ \\
\hline $\begin{array}{l}\text { Al-Khudairy } \\
2017^{12}\end{array}$ & $\begin{array}{l}\text { supplements of vit.C } \\
\text { alone, at any dose or } \\
\text { frequency } \\
\text { vs. } \\
\text { placebo / no } \\
\text { intervention }\end{array}$ & $\begin{array}{l}\text {-healthy adults } \\
\text { from GP } \\
\text {-adults at } \\
\text { moderate / high } \\
\text { risk of CVD }\end{array}$ & $\begin{array}{l}\text {-all-cause mortality } \\
\text {-CVD mortality } \\
\text {-non-fatal end } \\
\text { points }^{\mathrm{a}}\end{array}$ & $\begin{array}{c}8 \\
15,445\end{array}$ & $\begin{array}{l}\geq 3 \text { months } \\
3 \mathrm{~m}-8 \mathrm{y}\end{array}$ & $\begin{array}{l}14,641 \text { male physicians aged } \\
\geq 50 \text { with CVD risk factors } \\
(n=1), \text { men and women } \\
(n=6), \text { no specified gender } \\
(n=1)\end{array}$ & $\begin{array}{l}\text { reported but no } \\
\text { specified }(n=2)\end{array}$ \\
\hline $\begin{array}{l}\text { Bjelakovic } \\
2012^{13}\end{array}$ & $\begin{array}{l}\text { antioxidant } \\
\text { supplements ( carotene, } \\
\text { vit.A, vit.C, vit.E, and } \\
\text { selenium) at any dose, } \\
\text { duration and route of } \\
\text { administration } \\
\text { vs. } \\
\text { placebo / no } \\
\text { intervention }\end{array}$ & $\begin{array}{l}\text {-healthy adults } \\
\text {-adults from GP }\end{array}$ & -all-cause mortality & $\begin{array}{c}26 \\
215,900\end{array}$ & $\mathrm{NA}$ & $\mathrm{NA}$ & NS \\
\hline $\begin{array}{l}\text { Bjelakovic } \\
2014^{14}\end{array}$ & $\begin{array}{l}\text { supplements of vit.D, } \\
\text { alone or combined with } \\
\text { calcium, at any dose or } \\
\text { duration }^{\mathrm{b}} \\
\text { vs. } \\
\text { placebo / no } \\
\text { intervention }^{\mathrm{c}}\end{array}$ & $\begin{array}{l}\text {-healthy } \\
\text { adults from GP } \\
\text { irrespective of } \\
\text { serum vit.D }\end{array}$ & $\begin{array}{l}\text {-all-cause mortality } \\
\text {-CVD mortality } \\
-\mathrm{AE}\end{array}$ & $\begin{array}{c}48 \\
94,491\end{array}$ & $\mathrm{NA}$ & $\begin{array}{l}\text { healthy volunteers }(n=4) \text {, } \\
\text { postmenopausal women } \\
(n=9), \text { older people }(n=35)\end{array}$ & NA \\
\hline
\end{tabular}




\begin{tabular}{|c|c|c|c|c|c|c|c|}
\hline $\begin{array}{l}\text { Author } \\
\text { Year }\end{array}$ & $\begin{array}{l}\text { Intervention } \\
\text { Comparison }\end{array}$ & Population & Outcomes & $\begin{array}{c}\text { Studies } \\
\text { Participants }\end{array}$ & $\begin{array}{l}\text { Follow-up } \\
\text { Range }\end{array}$ & $\begin{array}{l}\text { Characteristics } \\
\text { of Participants }\end{array}$ & $\mathbf{A E}$ \\
\hline $\begin{array}{l}\text { Rees } \\
2013^{15}\end{array}$ & $\begin{array}{l}\text { supplements of } \\
\text { selenium alone } \\
\text { vs. } \\
\text { placebo / no } \\
\text { intervention }\end{array}$ & $\begin{array}{l}\text {-adults from GP } \\
\text {-adults at high } \\
\text { risk of CVD }\end{array}$ & $\begin{array}{l}\text {-all-cause mortality } \\
\text {-major CVD } \\
\text { events (CVD, MI, } \\
\text { non-fatal stroke, } \\
\text { revascularisation } \\
\text { procedures as } \\
\text { CABG or PTCA) } \\
\text {-CHD events (fatal } \\
\text { CHD, non-fatal MI) } \\
\text {-stroke events } \\
\text { (fatal and non-fatal } \\
\text { stroke, PAD) }\end{array}$ & $\begin{array}{c}12 \\
19,715\end{array}$ & $\begin{array}{l}\geq 3 \text { months } \\
1-2 w-12 y\end{array}$ & $\begin{array}{l}17,843 \text { men }(n=6) \text {, healthy } \\
\text { population }(n=3) \text {, prostate } \\
\text { cancer }(n=1) \text {, at high risk of } \\
\text { cancer }(n=2)\end{array}$ & $\begin{array}{l}\text { increased risk } \\
\text { d of DM2 }(1.06, \\
0.97-1.15 ; \mathrm{n}=3) \text { or } \\
\text { alopecia }(1.28,1.01- \\
.62) \text { and } 1-2 \text { grade } \\
\text { dermatitis }(1.17 \text {, } \\
1.0-1.35 ; \mathrm{n}=1)\end{array}$ \\
\hline $\begin{array}{l}\text { Flowers } \\
2014^{16}\end{array}$ & $\begin{array}{l}\text { supplements of co- } \\
\text { enzyme Q10 alone } \\
\text { vs. } \\
\text { placebo / no } \\
\text { intervention }\end{array}$ & $\begin{array}{l}\text {-adults aged } \geq 18 \\
\text { from GP } \\
\text {-adults at high } \\
\text { risk of CVD }\end{array}$ & $\begin{array}{l}\text {-all-cause mortality } \\
\text {-CVD mortality } \\
\text {-non-fatal } \\
\text { endpoints** } \\
\text {-AE }\end{array}$ & $\begin{array}{c}6 \\
218\end{array}$ & $\begin{array}{l}\geq 12 \text { weeks } \\
12-16 \mathrm{w}\end{array}$ & $\begin{array}{l}\text { men and women }(n=5) \text {, no } \\
\text { specified gender }(n=1) \text {, at } \\
\text { high risk of } \operatorname{CVD}(n=6 \text {; obese } \\
n=1 \text {, hypercholesterolemic } \\
n=4 \text {, hypertensive } n=1)\end{array}$ & NR \\
\hline $\begin{array}{l}\text { Hartley } \\
2015^{17}\end{array}$ & $\begin{array}{l}\text { supplements of vit.K } \\
\text { alone } \\
\text { vs. } \\
\text { placebo / no } \\
\text { intervention }\end{array}$ & $\begin{array}{l}\text {-healthy adults } \\
\text { aged } \geq 18 \text { from } \\
\text { GP } \\
\text {-adults at } \\
\text { moderate / high } \\
\text { risk of CVD }\end{array}$ & $\begin{array}{l}\text {-all-cause mortality } \\
\text {-CVD mortality } \\
\text {-non-fatal } \\
\text { endpoints } \\
\text {-AE }\end{array}$ & $\begin{array}{c}1 \\
60\end{array}$ & $\geq 3$ months & $\begin{array}{l}\text { healthy men and women } \\
\text { aged 40-6 with BMI=18.5-30 }\end{array}$ & NR \\
\hline $\begin{array}{l}\text { Fortmann } \\
2013^{18}\end{array}$ & $\begin{array}{l}\text { supplements of vitamins } \\
\text { (A, C, D, E) and minerals } \\
\text { (calcium, folic acid, } \\
\text { carotene, selenium) b,e } \\
\text { alone or combined } \\
\text { vs. } \\
\text { placebo / no } \\
\text { multivitamin }\end{array}$ & $\begin{array}{l}\text {-adults from GP } \\
\text { with no history } \\
\text { of CVD }\end{array}$ & $\begin{array}{l}\text {-all-cause mortality } \\
\text {-CVD events }\end{array}$ & $\begin{array}{l}24 \\
\text { NS }\end{array}$ & $\begin{array}{l}\geq 6 \text { months } \\
6 \mathrm{~m}-16 \mathrm{y} \\
\text { (most } \\
\text { studies } \geq \\
10 \mathrm{y} \text { ) }\end{array}$ & $\mathrm{NA}$ & $\begin{array}{l}\text { trivial } \mathrm{AE} \text { related to } \\
\text { vit. } \mathrm{A}(\mathrm{n}=1)\end{array}$ \\
\hline $\begin{array}{l}\text { Hooper } \\
2004^{19}\end{array}$ & $\begin{array}{l}\text { advice to reduce } \\
\text { sodium intake } \\
\text { vs. } \\
\text { placebo / no } \\
\text { intervention }\end{array}$ & $\begin{array}{l}\text {-healthy adults } \\
\text { aged } \geq 16 \text { with } \\
\text { normal / raised } \\
\text { blood pressure }\end{array}$ & $\begin{array}{l}\text {-total mortality } \\
\text {-combined CVD } \\
\text { events (fatal and } \\
\text { non-fatal MI, stroke, } \\
\text { angina, heart } \\
\text { failure, peripheral } \\
\text { vascular events, } \\
\text { sudden death, } \\
\text { non-scheduled CVD } \\
\text { interventions as } \\
\text { CABG, PTCA) }\end{array}$ & $\begin{array}{c}11 \\
3,514\end{array}$ & $\begin{array}{l}\geq 26 \text { months } \\
6 \mathrm{~m}-7 \mathrm{y}\end{array}$ & $\begin{array}{l}2,326 \text { healthy normotensive } \\
\text { adults mostly white, male, } \\
\text { mean age } 40(n=3), 387 \\
\text { untreated hypertensive } \\
\text { adults aged } 16-64(n=5), 801 \\
\text { treated hypertensive aged } \\
55-67(n=3)\end{array}$ & NS \\
\hline $\begin{array}{l}\text { Hartley } \\
2013^{20}\end{array}$ & $\begin{array}{l}\text { advice to increase } \\
\text { consumption of fruit } \\
\text { and vegetables (or } \\
\text { provision of them) } \\
\text { vs. } \\
\text { no /minimal } \\
\text { intervention }\end{array}$ & $\begin{array}{l}\text {-healthy adults } \\
\text { aged } \geq 18 \\
\text {-adults at high } \\
\text { risk of } \mathrm{CVD}^{\mathrm{f}}\end{array}$ & $\begin{array}{l}\text {-all-cause mortality } \\
\text {-CVD mortality } \\
\text {-non-fatal } \\
\text { endpoints }{ }^{\mathrm{a}} \\
\text {-AE }\end{array}$ & $\begin{array}{c}10 \\
1,730\end{array}$ & $\begin{array}{l}\geq 3 \text { months } \\
3-12 \mathrm{~m}\end{array}$ & $\begin{array}{l}\text { only women }(\mathrm{n}=1) \text {, healthy } \\
\text { post-menopausal women } \\
\text { with family breast cancer } \\
\text { history }(\mathrm{n}=1) \text {, women with } \\
\text { metabolic syndrome }(\mathrm{n}=2) \text {, } \\
\text { healthy adults }(\mathrm{n}=3) \text {, adults } \\
\text { with } \mathrm{LDL}=130 \text { - } 190 \mathrm{mg} / \mathrm{dL} \\
\text { and triglyceride } \leq 250 \mathrm{mg} / \\
\mathrm{dL}(\mathrm{n}=1) \text {, obese }(\mathrm{n}=1) \text {, with } \\
\text { colorectal adenomatous } \\
\text { polyps in the last } 5 \mathrm{y}(\mathrm{n}=1) \text {, } \\
\text { pre-metabolic or healthy } \\
(\mathrm{n}=1)\end{array}$ & $\begin{array}{l}\text { increased bowel } \\
\text { movements, bad } \\
\text { breath and body } \\
\text { odor }(n=3)\end{array}$ \\
\hline $\begin{array}{l}\text { Rees } \\
(2013)^{21}\end{array}$ & $\begin{array}{l}\text { Mediterranean dietary } \\
\text { pattern }^{g} \\
\text { vs. } \\
\text { no / minimal } \\
\text { intervention }^{\mathrm{h}} \\
\end{array}$ & $\begin{array}{l}\text {-adults from GP } \\
\text {-adults at high } \\
\text { risk of } C^{\mathrm{f}}\end{array}$ & $\begin{array}{l}\text {-all-cause mortality } \\
\text {-CVD mortality } \\
\text {-non-fatal } \\
\text { endpoints }{ }^{\mathrm{a}} \\
\text {-AE }\end{array}$ & $\begin{array}{c}11 \\
52,044\end{array}$ & $\begin{array}{l}\geq 3 \text { months } \\
3-8 y\end{array}$ & $\begin{array}{l}48,835 \text { women }(n=1), 49,185 \\
\text { healthy adults }(n=5)\end{array}$ & NR \\
\hline
\end{tabular}




\begin{tabular}{|c|c|c|c|c|c|c|c|}
\hline $\begin{array}{l}\text { Author } \\
\text { Year }\end{array}$ & $\begin{array}{l}\text { Intervention } \\
\text { Comparison }\end{array}$ & Population & Outcomes & $\begin{array}{c}\text { Studies } \\
\text { Participants }\end{array}$ & $\begin{array}{l}\text { Follow-up } \\
\text { Range }\end{array}$ & $\begin{array}{l}\text { Characteristics } \\
\text { of Participants }\end{array}$ & $\mathrm{AE}$ \\
\hline $\begin{array}{l}\text { Martin } \\
(2015)^{22}\end{array}$ & $\begin{array}{l}\text { advice to increase } \\
\text { consumption of nuts (or } \\
\text { provision of them) } \\
\text { vs. } \\
\text { no / minimal } \\
\text { intervention }\end{array}$ & $\begin{array}{l}\text {-adults from GP } \\
\text { with no history } \\
\text { of } \mathrm{CVD}^{\mathrm{e}}\end{array}$ & $\begin{array}{l}\text {-all-cause mortality } \\
\text {-CVD mortality } \\
\text {-non-fatal } \\
\text { endpoints } \\
\text {-AE }\end{array}$ & $\begin{array}{c}5 \\
435\end{array}$ & $\begin{array}{l}\geq 3 \text { months } \\
3-6 \mathrm{~m}\end{array}$ & $\begin{array}{l}\text { women, mean age }=37.4-54.3 \\
(\mathrm{n}=1)\end{array}$ & $\begin{array}{l}\text { allergic reaction } \\
\text { to nuts }(\mathrm{n}=1) \text {, } \\
\text { insignificant weight } \\
\text { gain }(\mathrm{n}=1)\end{array}$ \\
\hline $\begin{array}{l}\text { Hartley } \\
(2013)^{23}\end{array}$ & $\begin{array}{l}\text { intake of green / black } \\
\text { tea extracts or as a } \\
\text { beverage } \\
\text { vs. } \\
\text { no / minimal } \\
\text { intervention }^{\mathrm{h}} \text { / placebo }\end{array}$ & $\begin{array}{l}\text {-healthy adults } \\
\text {-adults at high } \\
\text { risk of } \mathrm{CVD}^{\mathrm{f}}\end{array}$ & $\begin{array}{l}\text {-all-cause mortality } \\
\text {-CVD mortality } \\
\text {-non-fatal } \\
\text { endpoints } \\
\text {-AE }\end{array}$ & $\begin{array}{c}11 \\
821\end{array}$ & $\begin{array}{l}\geq 6 \text { months } \\
3 \mathrm{~m}-2 \mathrm{y}\end{array}$ & $\begin{array}{l}\text { women }(n=4) \text {, } \\
\text { postmenopausal women } \\
\text { with osteopenia }(n=1) \text {, } \\
\text { breast cancer survivors } \\
(\mathrm{n}=1) \text {; either DM or two } \\
\text { other CVD risk factors } \\
(\mathrm{n}=1) \text {, up to moderate } \\
\text { hypercholesterolemia }(\mathrm{n}=2) \text {, } \\
\text { healthy participants }(\mathrm{n}=6)\end{array}$ & $\begin{array}{l}\text { prostate cancer, } \\
\text { appendicitis, } \\
\text { hospitalization } \\
\text { for influenza, and } \\
\text { retinal detachment } \\
\text { (n=5, unlikely } \\
\text { attributable to } \\
\text { intervention) }\end{array}$ \\
\hline $\begin{array}{l}\text { Hartley } \\
(2016)^{24}\end{array}$ & $\begin{array}{l}\text { advice to increase } \\
\text { dietary fibre } \\
\text { consumption (or } \\
\text { provision of fibre } \\
\text { supplements / high-fibre } \\
\text { foods) } \\
\text { vs. } \\
\text { no/minimal intervention }\end{array}$ & $\begin{array}{l}\text {-adults from GP } \\
\text {-adults at high } \\
\text { risk of CVD }\end{array}$ & $\begin{array}{l}\text {-all-cause mortality } \\
\text {-CVD mortality } \\
\text {-non-fatal } \\
\text { endpoints } \\
\text {-AE }\end{array}$ & $\begin{array}{c}23 \\
1,513\end{array}$ & $\begin{array}{l}\geq 3 \text { months } \\
\text { NS }\end{array}$ & $\begin{array}{l}\text { healthy participants }(n=4) \text {, } \\
\text { over weighted or obese } \\
(n=6) \text {, with clear signs of } \\
\text { metabolic syndrome }(n=3) \text {, } \\
\text { hypertensive }(n=3) \text {, women } \\
\text { with climacteric symptoms } \\
(n=1)\end{array}$ & $\begin{array}{l}\mathrm{n}=14 ; \\
\text { gastrointestinal } \\
\text { events (flatulence } \\
\text { and diarrhoea, } \\
\mathrm{n}=1 \text {; flatulence but } \\
\text { also constipation, } \\
\text { nausea, bloating } \\
\text { and diarrhoea, } \\
\mathrm{n}=7 \text { ); decrease of } \\
\text { antihypertensive } \\
\text { medication side- } \\
\text { effects scores } \\
\text { from baseline } \\
\text { observed only in } \\
\text { the intervention } \\
\text { group } \\
\text { (n=1) }\end{array}$ \\
\hline $\begin{array}{l}\text { Hooper } \\
(2004)^{25}\end{array}$ & $\begin{array}{l}\text { advice on diet } \\
\text { or provide a diet } \\
\text { to increase 3FA } \\
\text { by foodstuffs or } \\
\text { supplements (oil / } \\
\text { capsule) } \\
\text { vs. } \\
\text { placebo / no advice / } \\
\text { no supplementation / } \\
\text { usual diet }\end{array}$ & $\begin{array}{l}\text {-adults at any } \\
\text { risk of CVD }\end{array}$ & $\begin{array}{l}\text {-all-cause mortality } \\
\text {-fatal and non-fatal } \\
\text { CVD events (MI, } \\
\text { angina, stroke, } \\
\text { heart failure, } \\
\text { peripheral vascular } \\
\text { disease, sudden } \\
\text { death, CABG, } \\
\text { PTCA) } \\
\text {-cancer } \\
\text {-AE }\end{array}$ & $\begin{array}{c}48 \\
35,140\end{array}$ & $\begin{array}{l}\geq 6 \text { months } \\
11-48 \mathrm{~m}\end{array}$ & $\begin{array}{l}\text { at high }(\mathrm{n}=21), \text { moderate } \\
(\mathrm{n}=10) \text { or low risk }(\mathrm{n}=17) \text { of } \\
\text { CVD; male proportion } \geq 70 \% \\
(\mathrm{n}=24), 31-69 \%(\mathrm{n}=17), 0-30 \% \\
(\mathrm{n}=5), \text { not stated }(\mathrm{n}=2) ; \\
\text { mean ages in the } 30 \mathrm{~s}(\mathrm{n}=6), \\
40 \mathrm{~s}(\mathrm{n}=7), 50 \mathrm{~s}(\mathrm{n}=27), 60 \mathrm{~s} \\
(\mathrm{n}=4), 80 \mathrm{~s}(\mathrm{n}=3), \text { unclear } \\
(\mathrm{n}=1)\end{array}$ & $\begin{array}{l}\text { not significant } \\
\text { thrombophlebitis } \\
(1.59,0.72-3.51, \\
\mathrm{n}=1) \text {, urolithiasis } \\
(0.80,0.47-1.36, \\
\mathrm{n}=1) \text { and } \mathrm{DM}(0.87, \\
0.15-5.08, \mathrm{n}=2)^{\mathrm{d}}\end{array}$ \\
\hline $\begin{array}{l}\text { Al-Khudairy } \\
(2015)^{26}\end{array}$ & $\begin{array}{l}\text { advice on diet, } \\
\text { supplementation } \\
\text { or provide a diet to } \\
\text { increase / decrease 6FA } \\
\text { vs. } \\
\text { placebo / no advice / } \\
\text { no supplementation / } \\
\text { usual diet }\end{array}$ & $\begin{array}{l}\text {-healthy adults } \\
\text { from GP -adults } \\
\text { at moderate / } \\
\text { high risk of CVD }\end{array}$ & $\begin{array}{l}\text {-all-cause mortality } \\
\text {-CVD mortality } \\
\text {-non-fatal } \\
\text { endpoints } \\
\text {-AE }\end{array}$ & $\begin{array}{c}4 \\
664\end{array}$ & $\geq 6$ months & $\begin{array}{l}\text { over weighted or obese } \\
\text { healthy participants aged } \\
35-65(\mathrm{n}=1) \text { and } 40-70 \\
(\mathrm{n}=1) \text {, older men and post- } \\
\text { menopausal women aged } 45- \\
70(\mathrm{n}=1) \text {, adults aged } 23-58 \\
\text { with hypercholesterolemia } \\
(\mathrm{n}=1)\end{array}$ & NR \\
\hline $\begin{array}{l}\text { Ebrahim } \\
(2011)^{11}\end{array}$ & $\begin{array}{l}\text { health promotion } \\
\text { activity (counseling } \\
\text { or educational } \\
\text { intervention, } \\
\text { individually or by } \\
\text { group, with or without } \\
\text { pharmacological } \\
\text { treatment) to achieve } \\
\text { a behavioral change } \\
\text { altering } \geq 1 \text { CVD risk } \\
\text { factor } \\
\text { Parallel-group design }\end{array}$ & $\begin{array}{l}\text {-adults (aged } \\
\geq 35 \text { ) from GP } \\
\text {-adults from } \\
\text { occupational } \\
\text { groups } \\
\text {-adults with } \\
\text { specific risk } \\
\text { factors }^{\mathrm{f}}\end{array}$ & $\begin{array}{l}\text {-all-cause mortality } \\
\text {-fatal CHD and } \\
\text { stroke events } \\
\text {-non-fatal CHD (MI, } \\
\text { unstable angina, } \\
\text { CABG, PTCA, stroke } \\
\text { events) }\end{array}$ & $\begin{array}{c}55 \\
139,256\end{array}$ & $\begin{array}{l}\geq 6 \text { months. } \\
6 \mathrm{~m}-12 \mathrm{y} \\
\text { (median=1 y) }\end{array}$ & $\begin{array}{l}\text { mean age }=50 y \text {; adults } \geq 60 \\
(\mathrm{n}=2), \geq 75(\mathrm{n}=53) ; \text { patients } \\
\text { with } \mathrm{DM}(\mathrm{n}=5) \text { and with } \\
\text { hypertension }(\mathrm{n}=16)\end{array}$ & NS \\
\hline
\end{tabular}




\begin{tabular}{|c|c|c|c|c|c|c|c|}
\hline $\begin{array}{l}\text { Author } \\
\text { Year }\end{array}$ & $\begin{array}{l}\text { Intervention } \\
\text { Comparison }\end{array}$ & Population & Outcomes & $\begin{array}{c}\text { Studies } \\
\text { Participants }\end{array}$ & $\begin{array}{l}\text { Follow-up } \\
\text { Range }\end{array}$ & $\begin{array}{l}\text { Characteristics } \\
\text { of Participants }\end{array}$ & $\mathbf{A E}$ \\
\hline $\begin{array}{l}\text { Uthman } \\
(2015)^{27}\end{array}$ & $\begin{array}{l}\text { health promotion } \\
\text { intervention } \\
\text { (dietary advice, } \\
\text { increasing activity, } \\
\text { smoking cessation) } \\
\text { with or without } \\
\text { pharmacological } \\
\text { treatment to achieve } \\
\text { a behavioral change } \\
\text { altering } \geq 1 \text { CVD risk } \\
\text { factor } \\
\text { vs. } \\
\text { no intervention }\end{array}$ & $\begin{array}{l}\text {-adults from } \\
\text { the workforce, } \\
\text { including those } \\
\text { at high-risk (or } \\
\text { not) of CVD }\end{array}$ & $\begin{array}{l}\text {-CVD mortality } \\
\text {-non-fatal } \\
\text { endpoints } \\
\text {-AE }\end{array}$ & $\begin{array}{l}13 \\
\text { NS }\end{array}$ & $\begin{array}{l}\geq 6 \text { months } \\
6-30 \mathrm{~m} \\
\text { (mean }= \\
13.3 \mathrm{~m})\end{array}$ & $\begin{array}{l}\text { healthy or GP }(\mathrm{n}=2) \text {, high } \\
\text { CVD risk groups (pre- } \\
\text { and hypertensive, with } \\
\text { metabolic syndrome, obese, } \\
\text { with impaired glucose } \\
\text { regulation) ( } \mathrm{n}=11)\end{array}$ & NR \\
\hline $\begin{array}{l}\text { Seron } \\
(2014)^{28}\end{array}$ & $\begin{array}{l}\text { aerobic / resistance } \\
\text { exercise training } \\
\text { vs. } \\
\text { no exercise }\end{array}$ & $\begin{array}{l}\text {-people at high } \\
\text { CVD risk with } \\
\text { a Framingham } \\
\text { risk score } \geq 10 \% \\
\text { over } 10 \mathrm{y}\end{array}$ & $\begin{array}{l}\text {-CVD mortality } \\
\text {-all-cause mortality } \\
\text {-MI } \\
\text {-stroke }\end{array}$ & $\begin{array}{c}4 \\
823\end{array}$ & $\begin{array}{l}\geq 8 \text { weeks } \\
16 \mathrm{w}-6 \mathrm{~m}\end{array}$ & mean age $=52.6 y(n=4)$ & NR \\
\hline $\begin{array}{l}\text { Hartley } \\
(2015)^{29}\end{array}$ & $\begin{array}{l}\text { any style of Qigong } \\
\text { vs. } \\
\text { no / minimal } \\
\text { intervention (health } \\
\text { or physical activity } \\
\text { promotion without face- } \\
\text { to-face interaction) }\end{array}$ & $\begin{array}{l}\text {-healthy adults } \\
\text { aged } \geq 18 \text { years } \\
\text { from GP } \\
\text {-adults at high } \\
\text { risk of CVD }\end{array}$ & $\begin{array}{l}\text {-all-cause mortality } \\
\text {-CVD mortality } \\
\text {-non-fatal } \\
\text { endpoints } \\
\text {-AE }\end{array}$ & $\begin{array}{c}11 \\
1,369\end{array}$ & $\begin{array}{l}\geq 3 \text { months } \\
3 \mathrm{~m}-30 \mathrm{y}\end{array}$ & $\begin{array}{l}\text { men and women }(\mathrm{n}=5) \text {, men } \\
(\mathrm{n}=4), \text { not specified gender } \\
(\mathrm{n}=2) \text {; healthy participants } \\
(\mathrm{n}=2) \text {, elder with high blood } \\
\text { lipids }(\mathrm{n}=1) \text {, hypertensive } \\
\text { patients }(\mathrm{n}=8)\end{array}$ & NR \\
\hline $\begin{array}{l}\text { Hartley } \\
(2014)^{30}\end{array}$ & $\begin{array}{l}\text { any style of Tai chi } \\
\text { vs. } \\
\text { no / minimal } \\
\text { intervention }^{\mathrm{h}}\end{array}$ & $\begin{array}{l}\text {-healthy adults } \\
\text {-adults at high } \\
\text { risk of CVD }\end{array}$ & $\begin{array}{l}\text {-all-cause mortality } \\
\text {-CVD mortality } \\
\text {-non-fatal } \\
\text { endpoints } \\
\text {-AE }\end{array}$ & $\begin{array}{c}13 \\
1,520\end{array}$ & $\begin{array}{l}\geq 3 \text { months } \\
3-6 \mathrm{~m}\end{array}$ & $\begin{array}{l}\text { men and women }(n=9) \text {, only } \\
\text { women }(n=4) \text {; borderline } \\
(\mathrm{n}=1) \text { and essential }(\mathrm{n}=2) \\
\text { hypertensive, hypertensive } \\
\text { with liver and kidney } \\
\text { deficiency syndrome }(\mathrm{n}=1) \text {, } \\
\text { hypertensive }(\mathrm{n}=1) \text {, older } \\
\text { participants with high risk of } \\
\text { falling }(\mathrm{n}=1), \text { healthy people } \\
(903 \text { participants, } \mathrm{n}=7)\end{array}$ & NR \\
\hline $\begin{array}{l}\text { Hartley } \\
(2014)^{31}\end{array}$ & $\begin{array}{l}\text { any type of yoga with } \\
\text { postural exercises, } \\
\text { breathing control and } \\
\text { meditation } \\
\text { vs. } \\
\text { no / minimal } \\
\text { intervention }\end{array}$ & $\begin{array}{l}\text {-healthy adults } \\
\text {-adults at high } \\
\text { risk of CVD }\end{array}$ & $\begin{array}{l}\text {-all-cause mortality } \\
\text {-CVD mortality } \\
\text {-non-fatal } \\
\text { endpoints } \\
\text {-AE }\end{array}$ & $\begin{array}{c}11 \\
800\end{array}$ & $\begin{array}{l}\geq 12 \text { weeks } \\
14 w-8 m\end{array}$ & $\begin{array}{l}\text { only man }(\mathrm{n}=1) \text {, only women } \\
(\mathrm{n}=10) \text {, including healthy } \\
\text { premenopausal }(\mathrm{n}=1) \text {, with } \\
\text { metabolic syndrome }(\mathrm{n}=1) \text {, } \\
\text { obese postmenopausal } \\
(\mathrm{n}=1) \text {, with menstrual } \\
\text { irregularities }(\mathrm{n}=1) \text {; essential } \\
\text { hypertensive }(\mathrm{n}=1) \text {, males } \\
\text { with high cholesterol and } \\
\text { hypertension }(\mathrm{n}=1), \text { HIV- } \\
\text { infected participants ( } \mathrm{n}=1) \text {, } \\
\text { no yoga exercisers with } \\
\text { family history of DM2 and at } \\
\text { least one cardio- metabolic } \\
\text { risk factor }(\mathrm{n}=1) \text {, healthy } \\
\text { participants }(\mathrm{n}=3)\end{array}$ & NR \\
\hline $\begin{array}{l}\text { Hartley } \\
(2014)^{32}\end{array}$ & $\begin{array}{l}\text { transcendental } \\
\text { meditation } \\
\text { vs. } \\
\text { no / minimal } \\
\text { intervention }\end{array}$ & $\begin{array}{l}\text {-healthy adults } \\
\text {-adults at high } \\
\text { risk of CVD }\end{array}$ & $\begin{array}{l}\text {-all-cause mortality } \\
\text {-CVD mortality } \\
\text {-non-fatal } \\
\text { endpoints } \\
\text {-AE }\end{array}$ & $\begin{array}{c}4 \\
430\end{array}$ & $\begin{array}{l}\geq 3 \text { months } \\
\text { NS }\end{array}$ & $\begin{array}{l}\text { men and women }(\mathbf{n}=3) \text {, } \\
\text { only women }(\mathbf{n}=1) ; \text { healthy } \\
\text { participants (316 adults, } \\
\mathbf{n}=1) \text {, essential hypertensive } \\
(\mathbf{n}=1) \text {, older people }(\mathbf{n}=1)\end{array}$ & NR \\
\hline
\end{tabular}




\begin{tabular}{|c|c|c|c|c|c|c|c|}
\hline $\begin{array}{l}\text { Author } \\
\text { Year }\end{array}$ & $\begin{array}{l}\text { Intervention } \\
\text { Comparison }\end{array}$ & Population & Outcomes & $\begin{array}{c}\text { Studies } \\
\text { Participants }\end{array}$ & $\begin{array}{l}\text { Follow-up } \\
\text { Range }\end{array}$ & $\begin{array}{l}\text { Characteristics } \\
\text { of Participants }\end{array}$ & $\mathbf{A E}$ \\
\hline $\begin{array}{l}\text { Widmer } \\
(2015)^{33}\end{array}$ & $\begin{array}{l}\text { digital health } \\
\text { interventions b,e } \\
\text { (telemedicine, web- } \\
\text { based strategies, } \\
\text { email, mobile phone, } \\
\text { apps, text messaging, } \\
\text { monitoring sensors) } \\
\text { vs. } \\
\text { no active intervention } \\
\text { beyond usual care } \\
\text { following standard } \\
\text { guidelines }\end{array}$ & $\begin{array}{l}\text {-adults at any } \\
\text { risk of CVD }\end{array}$ & $\begin{array}{l}\text {-CVD events } \\
\text {-hospitalizations } \\
\text {-all-cause mortality }\end{array}$ & $\begin{array}{c}2 \\
1,055\end{array}$ & $\begin{array}{l}\geq 1 \text { month } \\
\text { NS }\end{array}$ & $\begin{array}{l}\text { hypertensive }(\mathrm{n}=1) \text {, over } \\
\text { weighted adults }(\mathrm{n}=1)\end{array}$ & NS \\
\hline $\begin{array}{l}\text { Krogsboll } \\
(2012)^{34}\end{array}$ & $\begin{array}{l}\text { general health checks } \\
\text { screening for } \geq 1 \\
\text { disease or risk factor } \\
\text { and in more than one } \\
\text { system by doctor, } \\
\text { nurse or other health } \\
\text { professional } \\
\text { vs. } \\
\text { control group }\end{array}$ & $\begin{array}{l}\text {-GP or } \\
\text { subgroups } \\
\text { (employees } \\
\text { from a company } \\
\text { aged <65, or } \\
\text { people with } \\
\text { specific risk } \\
\text { factors / } \\
\text { diseases) }\end{array}$ & $\begin{array}{l}\text {-all-cause mortality } \\
\text {-disease-specific } \\
\text { mortality } \\
\text {-morbidity }\end{array}$ & $\begin{array}{c}14 \\
182,880\end{array}$ & $4-22 y$ & no details of interest & NS \\
\hline
\end{tabular}

AE: adverse events; vit: vitamin; GP: General Population; CVD: cardiovascular disease; $m$ : months; $y$ : years; NA: not applicable as primary and secondary prevention data were mixed; NS: not studied; CHD: coronay heart disease; w: week; DM2: type 2 diabetes mellitus; NR: not reported; BMI: body mass index; $\omega$ FA: omega fatty acids.

a: myocardial infarction (MI), coronary artery bypass grafting (CABG), per cutaneous transluminal coronary angioplasty (PTCA), angina, angiographically defined coronary heart disease, stroke, carotid endarterectomy, and peripheral arterial disease (PAD); ${ }^{\circ}$ : only primary prevention information/ results provided; ${ }^{c}$ : calcium in the control group was included if equally used in the vitamin $\mathrm{D}$ groups of the trial; : : relative risk and confidence interval at $5 \%$; ${ }^{\text {e: }}$ only information about random clinical trials; ${ }^{\text {f: }}$ studies excluded if $\geq 25 \%$ participants had CVD; s: consumption of at least two of the following dietary factors: high monounsaturated/saturated fat ratio (use of olive oil as main cooking ingredient), low to moderate red wine consumption, high consumption of legumes, high consumption of grains and cereals, high consumption of fruits and vegetables, low consumption of meat and meat products and increased consumption of fish, moderate consumption of milk and dairy products; ${ }^{\text {h}}:$ as leaflets with no person-to-person interaction or reinforcement; ${ }^{i}:$ studies excluded if $\geq 25 \%$ participants had diagnosed CVD or DM2.

for vitamin $\mathrm{K}$ supplements ${ }^{20}$ and 215,900 participants for antioxidant supplementation $^{13}$. Only four reviews included less than 500 participants ${ }^{16,17,22,32}$. The number of trials per review ranged between one ${ }^{17}$ and $55^{19}$. Most studies were carried out in developed countries, except one conducted in low- and middle-income countries ${ }^{27}$. Most reviews defined a minimum follow up time (between three and six months) for RCTs to be included; the maximum follow up time was up to 20-30 years ${ }^{29}$.

Low risk of bias for nine of the eleven items included in the AMSTAR checklist (Appendix 1) was showed by all studies, exception made by five studies whose authors did not evaluate the risk of publication bias and/or report the results ${ }^{18-20,22,28,34}$ (item 10). Eleven studies did not report outcomes of interest, preventing to combine results and, thus, scoring was not applicable.

\section{Deaths from any cause}

Several interventions showed some risk reduction in all-cause mortality ${ }^{14,15,18,19,27,34,47}$ : reduce dietary salt ${ }^{19}$, selenium supplementation $^{15,18}$, folic acid + vitamin D + calcium supplementation $^{18}$, general health checks ${ }^{34}$, vitamin D supplementations ${ }^{14}$ and practice of Qigong ${ }^{29}$, but only the two last reached statistical significance (Table 2). The antioxidant supplements ${ }^{13}$ showed a significant increase of $5 \%$ [95\% CI 2-8\%] for all-cause mortality risk.

\section{Cardiovascular mortality}

Both multiple risk factor interventions ${ }^{11}$ and Qigong practice ${ }^{29}$ showed pooled significant reductions in stroke mortality (risk reduction 25\% [95\% CI 5-40\%] and 45\% [95\% CI 26-58\%], respectively) (Table 3). 
Table 2. Effect of the interventions on all-cause mortality

\begin{tabular}{|c|c|c|c|c|}
\hline Intervention & $\begin{array}{c}\text { Studies }^{*} \\
\mathbf{N}\end{array}$ & $\begin{array}{c}\text { Events } \\
\text { N (\%) }\end{array}$ & $\begin{array}{c}\text { Effect } \\
\text { RR }(95 \% \mathrm{CI})\end{array}$ & $\begin{array}{l}I^{2} \\
(\%)\end{array}$ \\
\hline \multirow{3}{*}{ Antioxidant supplementation ${ }^{13}$} & 19 & $21,334(12.0)$ & $1.03(0.97-1.08)^{\mathrm{a}, \mathrm{b}}$ & 43.0 \\
\hline & 19 & $21,334(12.0)$ & $1.05(1.02-1.08)^{\mathrm{a}, \mathrm{c}}$ & 46.9 \\
\hline & 7 & $2,479(6.6)$ & $0.93(0.84-1.03)^{\mathrm{d}, \mathrm{b}}$ & 0 \\
\hline Vitamin A supplementation ${ }^{18}$ & 1 & $115(5.0)$ & $1.15(0.81-1.65)$ & NA \\
\hline \multirow{2}{*}{ Vitamin C supplementation ${ }^{12,18}$} & 1 & - & $\mathrm{HR}=1.07(0.97-1.18)^{\mathrm{e}}$ & NA \\
\hline & 2 & $1,663(11.2)$ & $1.06(0.97-1.16)$ & $\mathrm{NI}$ \\
\hline Vitamin D supplementation ${ }^{14}$ & 48 & $11,955(12.7)$ & $0.97(0.9-0.99)^{b}$ & 0 \\
\hline Vitamin D + Calcium supplementation ${ }^{18}$ & 1 & $1,551(4.3)$ & $0.92(0.83-1.01)$ & NA \\
\hline Calcium supplementation ${ }^{18}$ & 3 & $1,827(23.8)$ & $1.04(0.96-1.12)$ & $\mathrm{NI}$ \\
\hline Folic acid supplementation ${ }^{18}$ & 1 & $29(2.8)$ & $0.52(0.24-1.10)$ & NA \\
\hline Selenium supplementation ${ }^{15,18}$ & 2 & $1,336(7.2)$ & $0.97(0.88-1.08)^{\mathrm{c}}$ & 0 \\
\hline Omega 3 fatty acids ${ }^{25}$ & 44 & $1,995(5.5)$ & $0.87(0.73-1.03)$ & 0 \\
\hline Advice to reduce dietary salt ${ }^{19}$ & 4 & $17(0.7)$ & $0.90(0.36-2.24)^{\mathrm{a}}$ & 0 \\
\hline Qigong $^{29}$ & 2 & $147(28.8)$ & $0.54(0.39-0.90)^{\mathrm{a}, \mathrm{f}}$ & 0 \\
\hline General health checks ${ }^{34}$ & 9 & $11,940(7.7)$ & $0.99(0.95-1.03)^{\mathrm{c}}$ & 0 \\
\hline Multiple risk factor interventions ${ }^{11}$ & 14 & $2,511(1.8)$ & $\mathrm{OR}=1.00(0.96-1.05)^{\mathrm{c}}$ & 62 \\
\hline
\end{tabular}

${ }^{*}$ : number of included studies providing information of interest; RR: relative risk; $\mathrm{I}^{2}$ : Higgins' heterogeneity; a: primary prevention trials with low risk of bias; ${ }^{b}$ : random effects model analysis; c: fixed-effect model analysis; ${ }^{\text {d: primary }}$ prevention trials with a high risk of bias; e: data from the clinical trial Physicians Health Study II (men aged $\geq 50$ ); NA: not applicable; HR: hazard ratio; NI: No information; ${ }^{\mathrm{f}}:$ this data was calculated by the authors of the present review as it was not provided in the original review; OR: odds ratio. Effects in bold are statistically significant.

Table 3. Effect of the interventions on cardiovascular mortality

\begin{tabular}{|c|c|c|c|c|}
\hline Intervention & $\begin{array}{c}\text { Studies }^{*} \\
\mathbf{N}\end{array}$ & $\begin{array}{c}\text { Events } \\
\text { N (\%) }\end{array}$ & $\begin{array}{c}\text { Effect } \\
\text { RR }(95 \% \text { CI) }\end{array}$ & $\begin{array}{l}I^{2} \\
\text { (\%) }\end{array}$ \\
\hline Vitamin C supplementation ${ }^{12}$ & 1 & - & $\mathrm{HR}=1.02(0.85-1.22)^{\mathrm{a}}$ & $\mathrm{NA}$ \\
\hline Selenium supplementation ${ }^{15}$ & 2 & $342(1.9)$ & $0.97(0.79-1.20)$ & 44 \\
\hline Omega 3 fatty acids ${ }^{25}$ & 14 & $1,418(4.3)$ & $0.85(0.68-1.06)$ & 0 \\
\hline Advice to reduce dietary salt ${ }^{19}$ & 2 & $17(2.3)$ & $0.82(0.56-1.21)^{\mathrm{b}}$ & 0 \\
\hline \multirow{2}{*}{ Mediterranean dietary pattern ${ }^{21}$} & \multirow{2}{*}{1} & \multirow{2}{*}{-} & $\mathrm{HR}=1.01(0.81-1.27)^{\mathrm{c}, \mathrm{d}}$ & NA \\
\hline & & & $\mathrm{HR}=0.94(0.65-1.35)^{\mathrm{d}, \mathrm{e}}$ & NA \\
\hline Qigong $^{29}$ & 2 & $101(19.8)$ & $0.55(0.42-0.74)^{b, e, f}$ & 1 \\
\hline General health checks ${ }^{34}$ & 8 & $4,567(29.6)$ & $1.03(0.91-1.17)^{g}$ & 64 \\
\hline \multirow{2}{*}{ Multiple risk factor interventions ${ }^{11}$} & 11 & $901(2.2)$ & $\mathrm{OR}=0.99(0.92-1.07)^{\mathrm{c}, \mathrm{g}}$ & 32 \\
\hline & 7 & $342(0.6)$ & OR=0.75 (0.60-0.95) $)^{\mathrm{e}, \mathrm{g}}$ & 0 \\
\hline
\end{tabular}

*: number of included studies providing information of interest; RR: relative risk; $\mathrm{I}^{2}$ : Higgins' heterogeneity; HR: hazard ratio; ${ }^{a}$ : data from the clinical trial Physicians Health Study II (men aged $\geq 50$ ); NA: not applicable; ${ }^{\text {b: }}$ random effects

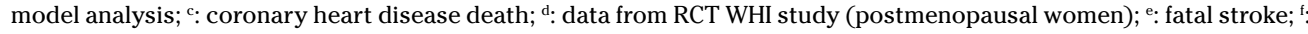
this data was calculated by the authors of the present review as it was not provided in the original review; $\mathrm{g}$ : fixed-effect model analysis. Parameters in bold are statistically significant. 


\section{Cardiovascular events}

The effect of interventions on CVD events, either a combined outcome of fatal and non-fatal events, or only non-fatal events are showed in table 4. A significant risk reduction of $16 \%$ [95\% CI 2-23\%] was found in multifactorial individual lifestyle interventions ${ }^{11}$, despite substantial between-study heterogeneity. Subgroup analysis showed that the combined event relative risk was $0.71(\mathrm{p}<0.05)$ in patients with either hypertension or diabetes, while there was no significant effect on patients without co-morbidity.

Qigong ${ }^{29}$ showed a significant reduction of stroke incidence (44\% [95\% CI 17-62\%]).

Increased consumption (dietary or supplemental) of omega 3 fatty acids, showed a significant risk reduction of heart failure of $49 \%(95 \% \text { CI } 15-69 \%)^{25}$.

\section{Adverse events}

Eight of the 24 included reviews reported adverse events related with the interventions: vitamin $\mathrm{C}^{12}$, selenium ${ }^{15}$ and mineral and multivitamin ${ }^{18}$ supplements, increased consumption of fruits and vegetables ${ }^{20}$, and also in nut ${ }^{22}$, green and black tea ${ }^{23}$ and dietary fibre ${ }^{24}$ consumption (Table 1). Al-Khudairy et $\mathrm{al}^{12}$ reported one RCT describing death, serious adverse events and adverse event without any further detail. In the other reviews, adverse events were a minor problem and/or the difference between the intervention and the control group was not statistically significant. In two reviews ${ }^{18,21}$, the data regarding adverse events were aggregated, thus primary was indistinguishable from secondary prevention.

\section{DISCUSSION}

This umbrella review provides information about diverse interventions for the primary prevention of CVD events and mortality. Our work constitutes the first comprehensive and systematic summary of diverse non-pharmacological interven- tions based on the umbrella review methodology. We included twenty-four reviews concerning non-pharmacological interventions; four of the thirteen reviews reporting CVD events or mortality found a significant reduction on them ${ }^{11,14,25,29}$.

Ebrahim et $\mathrm{al}^{11}$ found that individual lifestyle interventions significantly reduced stroke-related mortality and any CVD event (fatal and non-fatal). These interventions pursued a behavioural change in order to alter more than one cardiovascular risk factor (i.e. diet, reduce blood pressure, smoking, total blood cholesterol or increase physical activity) by means of counselling or educational interventions, with or without pharmacological treatments. They were commonly provided by a variety of health professionals (physicians, nurses, nutritionists, dieticians, personal trainers, cooks, psychotherapists and physiotherapists). Focusing multicomponent lifestyle interventions (dietary advice, smoking cessation, stress reduction, physical exercise, etc.) on individual risk factors -taking account of the characteristics of the patients and, furthermore, targeting at those most likely to benefit (for example, those with hypertension or diabetes)- is very important, as these interventions seem to not benefit patients without the risk factors ${ }^{11}$.

Bjelakovic $^{14}$ found that vitamin D supplementation at any dose, for any duration, in monotherapy or in combination with calcium, administrated enteral or parenterally, significantly reduced all-cause mortality.

Hooper $^{26}$ found that omega-3 fatty acid intervention (dietary supplementation, a provided diet or advice on diet such that the product orally consumed as oil or capsule or as food stuffs had an omega 3 fat content of at least $10 \%$ of the total fat content) significantly reduced the risk of heart failure.

Finally, Hartley ${ }^{29}$ found that Qigong significantly reduced stroke incidence, allcause mortality and stroke-related mortality. Qigong is defined by the National Qigong Association $^{35}$ as an ancient Chinese health care system that integrates physical postures, breathing techniques and focused intention.

Systematic reviews included in this umbrella review were generally well conduct- 
Table 4. Effect of the interventions on all cardiovascular events (fatal and/or non-fatal)

\begin{tabular}{|c|c|c|c|c|}
\hline Intervention & $\begin{array}{c}\text { Studies* } \\
\mathbf{N}\end{array}$ & $\begin{array}{c}\text { Events } \\
\mathrm{N}(\%)\end{array}$ & $\begin{array}{c}\text { Effect } \\
\text { RR (95\%Cl) }\end{array}$ & $\begin{array}{l}I^{2} \\
\text { (\%) }\end{array}$ \\
\hline Beta-Carotene supplementation ${ }^{18}$ & 2 & $2,157(3.5)$ & $1.01(0.93-1.09)^{\mathrm{a}}$ & $\mathrm{NI}$ \\
\hline \multirow{5}{*}{ Vitamin C supplementation ${ }^{12,18}$} & \multirow{4}{*}{1} & \multirow{4}{*}{-} & $\mathrm{HR}=1.04(0.87-1.24)^{\mathrm{b}, \mathrm{c}}$ & NA \\
\hline & & & $\mathrm{HR}=0.96(0.86-1.07)^{\mathrm{b}, \mathrm{d}}$ & NA \\
\hline & & & $\mathrm{HR}=0.93(0.84-1.03)^{\mathrm{b}, \mathrm{e}}$ & NA \\
\hline & & & $\mathrm{HR}=0.89(0.74-1,07)^{\mathrm{b}, \mathrm{f}}$ & NA \\
\hline & 1 & $1,245(8.5)$ & $0.99(0.89-1.10)^{\mathrm{a}}$ & NA \\
\hline Vitamin D supplementation ${ }^{18}$ & 2 & $1,682(21.1)$ & $0.94(0.87-1.02)^{\mathrm{a}}$ & NI \\
\hline Vitamin D + Calcium supplementation ${ }^{18}$ & 1 & $3,642(10.1)$ & $1.01(0.95-1.07)^{\mathrm{a}}$ & NA \\
\hline Calcium supplementation $^{18}$ & 1 & $96(10.3)$ & $1.09(0.75-1.60)^{\mathrm{a}}$ & NA \\
\hline Vitamin E supplementation ${ }^{18}$ & 3 & $4,328(6.0)$ & $0.97(0.92-1.03)^{\mathrm{a}}$ & $\mathrm{NI}$ \\
\hline \multirow{5}{*}{ Selenium supplementation ${ }^{15,18}$} & 2 & $2,036(11.0)$ & $0.96(0.89-1.04)^{\mathrm{g}, \mathrm{h}}$ & 0 \\
\hline & 1 & $165(1.0)$ & $0.79(0.58-1.07)^{\mathrm{i}}$ & NA \\
\hline & 1 & $22(0.1)$ & $0.99(0.43-2,29)^{\mathrm{j}}$ & NA \\
\hline & 1 & $197(1.1)$ & $0.90(0.62-1.32)^{\mathrm{k}}$ & NA \\
\hline & 2 & $2,329(12.6)$ & $1.03(0.95-1.11)^{\mathrm{a}}$ & 0 \\
\hline \multirow{8}{*}{ Omega 3 fatty acids ${ }^{25}$} & 31 & $2,628(7.5)$ & $0.95(0.82-1.12)^{\mathrm{a}, \mathrm{l}}$ & 65 \\
\hline & 38 & $390(84.0)$ & $0.86(0.60-1.25)^{\mathrm{c}}$ & 48 \\
\hline & 25 & $648(3.8)$ & $1.03(0.70-1.50)^{\mathrm{m}}$ & 51 \\
\hline & 25 & $565(3.3)$ & $0.77(0.59-1.02)^{\mathrm{e}}$ & 66 \\
\hline & 26 & $243(0.7)$ & $1.17(0.91-1.51)^{\mathrm{f}}$ & 0 \\
\hline & 20 & $54(0.7)$ & $0.51(0.31-0.85)^{\mathrm{n}}$ & 0 \\
\hline & 17 & $11(1.0)$ & $0.26(0.07-1.06)^{\circ}$ & 0 \\
\hline & 37 & $416(2.2)$ & $0.85(0.49-1.48)^{p}$ & 72 \\
\hline Advice to reduce dietary salt ${ }^{19}$ & 2 & $92(12.3)$ & $0.82(0,56-1,21)^{1, q}$ & 0 \\
\hline \multirow{4}{*}{ Mediterranean dietary pattern ${ }^{21}$} & \multirow{4}{*}{1} & \multirow{4}{*}{-} & $\mathrm{HR}=0.96(0.89-1.03)^{\mathrm{a}, \mathrm{r}}$ & NA \\
\hline & & & $\mathrm{HR}=0.91(0.8-1.04)^{\mathrm{m}, \mathrm{r}}$ & NA \\
\hline & & & $\mathrm{HR}=0.91(0.82-1.01)^{\mathrm{d}, \mathrm{r}}$ & NA \\
\hline & & & $\mathrm{HR}=1.04(0.90-1.19)^{\mathrm{i}, \mathrm{r}}$ & NA \\
\hline Qigong $^{29}$ & 1 & $80(26.1)$ & $0.56(0.38-0.83)^{f}$ & NA \\
\hline Health promotion interventions $s^{27}$ & 1 & $6(2.6)$ & $0,57(0.11-3.07)$ & NA \\
\hline Digital Health Interventions ${ }^{33}$ & 2 & $42(4.0)$ & $1,21(0.58-2.58)^{\mathrm{a}}$ & 15 \\
\hline Multiple risk factor interventions ${ }^{11}$ & 9 & $10,071(8.3)$ & $0.84(0.73-0.98)^{\mathrm{a}, \mathrm{h}}$ & 82 \\
\hline
\end{tabular}

*: number of included studies providing information of interest; RR: relative risk; $I^{2}$ : Higgins' heterogeneity; HR: hazard ratio; a: all cardiovascular events (fatal and non-fatal); NI: not informed; NA: not applicable; ${ }^{\text {b: }}$ data from the clinical trial Physicians Health Study II (men aged $\geq 50$ ); c: myocardial infarction; d: revascularization; e: angina; $:$ stroke; ${ }^{\text {: }}$ non-fatal

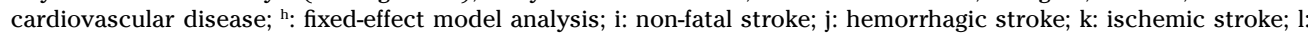
random effects model analysis; m: non-fatal myocardial infarction; n: heart failure; o: peripheral vascular events; p: sudden death; q: cardiovascular morbidity; r: data from RCT WHI study (postmenopausal women). Parameters in bold are statistically significant. 
ed. Nonetheless, in most reviews authors called for caution in extrapolating results, as a high risk of bias in some of the RCTs was noted due to participants at varying levels of risk, different intensities interventions, lack of statistical power and insufficient length of follow-up.

Conducting RCTs in primary prevention can be challenging, and perhaps this explains scarcity of interventions and show evidence of benefit on CVD outcomes. The studies are targeted at a general population, which includes people at low risk. The interventions generally have a behavioural element, which requires a certain degree of collaboration from participants to maintain the healthy behaviour over long periods. A long follow-up is required to provide enough statistical power for CVD outcomes. The authors of the Qigong review $^{29}$ realised that the included trials were originally designed to study outcomes over one year, but it was unclear from the RCTs whether both Qigong was continuously practiced over the whole follow up and if randomization was preserved in the analysis of the RCTs over the whole follow up. Other reviews have found more positive results on intermediate, short term outcomes such as cholesterol level and high blood pressure ${ }^{13,16,17,19,22,24,26-28,30}$.

The effectiveness of vitamin $\mathrm{D}$ in reducing all-cause mortality concur with the results found by Theodoratou et $\mathrm{al}^{36}$ who conducted an umbrella review across systematic reviews and meta-analyses of observational studies of 25-hydroxyvitamin D or 1,25-dihydroxyvitamin D concentrations in plasma, along with randomised controlled trials where the intervention was vitamin D supplementation for multiple health outcomes. The results of meta-analysis showed some insignificant risk reduction in CVD and mortality outcomes across randomised controlled trials with vitamin D supplementation, while a statistically significant risk reduction was seen in the observational studies about vitamin D concentrations in plasma.

Apart from vitamin D, no other supplements showed a benefit for cardiovascular prevention. The supplements are marketed in several countries, and many people take these pills believing that it will improve their health ${ }^{37}$. Nevertheless the effectiveness and safety of these products ought to be further evaluated. Antioxidant supplements may even increase the risk of overall mortality ${ }^{13}$. Furthermore, other meta-analyses have associated supplements with increased risk of some cancers ${ }^{37}$.

Limitations of this review are: only reviews in English and Spanish were included, and grey literature (e.g. non peer reviewed papers in unconventional information channels) was not searched; thus, we may therefore have missed some relevant studies. The strengths are: the review was conducted over several databases, and the overall risk of bias is likely to be very low as the majority of the included reviews are from Cochrane collaboration.

A limitation of the umbrella review methodology may be that we have assessed the quality of the systematic reviews using the risk of bias tool, not the quality of the individual studies within these reviews. Furthermore, the trials included in each systematic review can be quite heterogeneous in population (the Women's Health Initiative about diet in postmenopausal wom$\mathrm{en}^{20}$, or the Physicians Health Study II about vitamin $\mathrm{C}$ supplementation in male physicians aged 50 years or older ${ }^{12}$ ), causing that observed results may not be transferable to the general population. More research should be conducted to assess whether the effect of treatment varies according to population characteristics such as gender ${ }^{38}$, age or social class ${ }^{39}$. Longer-term monitoring of patients is needed to assess CVD.

Finally, the results of this review should be carefully interpreted as most studies compare non-pharmacological interventions for primary prevention against usual care, which can include recommended pharmacological treatment in higher risk patients (e.g. statins and/or antihypertensive, etc.). This means that these trials are measuring the combined effect of non-pharmaceutical interventions alongside standard pharmacological practice, rather than considering these interventions as an alternative to pharmacology. More research 
is also recommended to evaluate combinations of different interventions.

These results inform to future guidelines and clinical practice for intervention related to primary prevention of CVD. From the list of putative interventions to improve the cardiovascular health of patients in primary prevention, four of them have been shown to be effective, with minor adverse events if any. Other non-pharmacological interventions did not achieve statistical significance for CVD prevention, but did achieve significant improvements in other intermediate outcomes, such as blood pressure and lipid levels. Hence, further research is required to establish if these interventions, alone or in combination, translate into definite longterm health benefits. Given the limited evidence to date, there is therefore a need for well-conducted RCT with higher-quality design focused on long term outcomes such as CVD, as well as intermediate and surrogate endpoints, involving a long-term monitoring of non-pharmacological interventions on primary prevention to assess their effects on CVD and mortality. The role of the healthcare professional in engaging and communicating with patients also deserves further attention.

\section{Acknowledgements}

The authors are grateful to Simon Thompson, Stephen Kaptoge, for advice and support, and Camila Higueras, librarian EASP.

\section{REFERENCES}

1. RoBINSON JG. Starting primary prevention earlier with statins. Am J Cardiol 2014; 114:1437-1442.

2. NICE guidelines could put 12 million UK adults on statins. BMJ 2017; 358: j3674.

3. GoldACRE B. Mass treatment with statins. BMJ 2014; 349: g4745.

4. Townsend N, Nichols M, Scarborough P, Rayner M. Cardiovascular disease in Europe 2015: epidemiological update. Eur Heart J 2015; 36: 26732674.

5. WHO, United States Centers for Disease Control and Prevention (US CDC) Global Hearts Initiative. Working together to beat cardiovascular disease. [Access on: 20/03/2017] Available from http://www.who.int/cardiovascular_diseases/global-hearts/en/
6. The JoAnna Briggs Institute. Joanna Briggs Institute Reviewers' Manual: 2014 edition. Supplement. Adelaide: The Joanna Briggs Institute 2014. [Accessed 01/09/2017]. Available from: http://joannabriggs.org/assets/docs/sumari/ ReviewersManual-Methodology-JBI_Umbrella\%20Reviews-2014.pdf

7. Smith V, Devane D, Begley CM, Clarke M. Methodology in conducting a systematic review of systematic reviews of healthcare interventions. BMC Med Res Methodol 2011; 11: 15. DOI: 10.1186/1471-2288-11-15.

8. Shea BJ, Grimshaw JM, Wells GA, Boers M, ANDERSSON N, HAMEL C et al. Development of AMSTAR: a measurement tool to assess the methodological quality of systematic reviews. BMC Med Res Methodol 2007; 7: 10. DOI: 10.1186/1471-2288-7-10.

9. Higgins JP, Thompson SG. Quantifying heterogeneity in a meta-analysis. Statistics in Medicine 2002; 21: 1539-1558.

10. Ketola E, SiPILÄ R, MÄKELÄ M. Effectiveness of individual lifestyle interventions in reducing cardiovascular disease and risk factors. Ann Med 2000; 32: 239-251.

11. Ebrahim S, Taylor F, Ward K, Beswick A, Burke M, Davey SMith G. Multiple risk factor interventions for primary prevention of coronary heart disease. Cochrane Database Syst Rev 2011; 1. CD001561. DOI: 10.1002/14651858. CD001561.pub3.

12. Al-Khudairy L, Flowers N, Wheelhouse R, Ghannam O, Hartley L, Stranges $\mathrm{S}$ et al. Vitamin C supplementation for the primary prevention of cardiovascular disease. Cochrane Database Syst Rev 2017; 3. CD011114. DOI: 10.1002/14651858.CD011114.pub2.

13. Bjelakovic G, Nikolova D, Gluud LL, Simonetti RG, GLuUd C. Antioxidant supplements for prevention of mortality in healthy participants and patients with various diseases. Cochrane Database Syst Rev 2012: 3: CD007176. doi: 10.1002/14651858.CD007176.pub2.

14. Bjelakovic G, GluUd LL, Nikolova D, Whitfield K, WetTERSLEV J, SimONETti RG et al. Vitamin D supplementation for prevention of mortality in adults. Cochrane Database Syst Rev 2014; 1. CD007470. DOI: 10.1002/14651858.CD007470.pub2.

15. Rees K, Hartley L, Day C, Flowers N, Clarke A, STRANGes S. Selenium supplementation for the primary prevention of cardiovascular disease. Cochrane Database Syst Rev 2013; 1. CD009671. DOI: 10.1002/14651858.CD009671. pub2.

16. Flowers N, Hartley L, Todkill D, Stranges S, Rees K. Co-enzyme Q10 supplementation for the primary prevention of cardiovascular disease. Cochrane Database Syst Rev 2014; 12. CD010405. DOI: 10.1002/14651858.CD010405. pub2. 
17. Hartley L, Clar C, Ghannam O, Flowers N, Stranges $\mathrm{S}$, REES K. Vitamin K for the primary prevention of cardiovascular disease. Cochrane Database Syst Rev 2015; 9. CD011148. DOI: 10.1002/14651858. CD011148.pub2.

18. Fortmann SP, Burda BU, Senger CA, Lin JS, Whitlock EP. Vitamin, Mineral, and Multivitamin Supplements for the Primary Prevention of Cardiovascular Disease and Cancer: A Systematic Evidence Review for the U.S. Ann Intern Med 2013; 159: 824 834.

19. Hooper L, Bartlett C, Davey Smith G, Ebrahim S. Advice to reduce dietary salt for prevention of cardiovascular disease. Cochrane Database Syst Rev 2004; 1. CD003656. DOI: 10.1002/14651858. CD003656.pub2.

20. Hartley L, Igbinedion E, Holmes J, Flowers N, ThoroGOOD M, CLARKE A. Increased consumption of fruit and vegetables for the primary prevention of cardiovascular diseases. Cochrane Database Syst Rev 2013; 6. CD009874. DOI: 10.1002/14651858. CD009874.pub2.

21. Rees K, Hartley L, Flowers N, Clarke A, Hooper L, THorogood $\mathrm{M}$ et al. 'Mediterranean' dietary pattern for the primary prevention of cardiovascular disease. Cochrane Database Syst Rev 2013; 8. CD009825.

22. Martin N, Germanò R, Hartley L, Adler AJ, Rees K. Nut consumption for the primary prevention of cardiovascular disease. Cochrane Database Syst Rev 2015; 9. CD011583. DOI: 10.1002/14651858. CD011583.pub2.

23. Hartley L, Flowers N, Holmes J, Clarke A, Stranges $\mathrm{S}$, HoOper $\mathrm{L}$ et al. Green and black tea for the primary prevention of cardiovascular disease. Cochrane Database Syst Rev 2013; 6.CD009934. DOI: 10.1002/14651858.CD009934.pub2.

24. Hartley L, May MD, Loveman E, Colquitt JL, Rees K. Dietary fibre for the primary prevention of cardiovascular disease. Cochrane Database Syst Rev 2016; 1.CD011472. DOI: 10.1002/14651858. CD011472.pub2.

25. Hooper L, Thompson RL, Harrison RA, Summerbell CD, Moore H, Worthington HV et al. Omega 3 fatty acids for prevention and treatment of cardiovascular disease. Cochrane Database Syst Rev 2004; 4.CD003177. DOI: 10.1002/14651858.CD003177. pub2.

26. Al-Khudairy L, Hartley L, Clar C, Flowers N, HoopER L, REES K. Omega 6 fatty acids for the primary prevention of cardiovascular disease. Cochrane Database Syst Rev 2015; 11.CD011094. DOI: 10.1002/14651858.CD011094.pub2.

27. Uthman OA, Hartley L, Rees K, Taylor F, Ebrahim S, ClaRKE A. Multiple risk factor interventions for primary prevention of cardiovascular disease in low- and middle-income countries. Cochrane Database Syst Rev 2015; 8. DOI: 10.1002/14651858. CD011163.pub2.
28. Seron P, Lanas F, Pardo-Hernandez H, Bonfill Cosp X. Exercise for people with high cardiovascular risk. Cochrane Database Syst Rev 2014; 8.CD009387. DOI: 10.1002/14651858.CD009387.pub2.

29. Hartley L, LeE MS, Kwong JSW, Flowers N, TodKILL D, ERnST E et al. Qigong for the primary prevention of cardiovascular disease. Cochrane Database Syst Rev 2015; 6.CD010390. DOI: 10.1002/14651858. CD010390.pub2.

30. Hartley L, Flowers N, Lee MS, Ernst E, Rees K. Tai chi for primary prevention of cardiovascular disease. Cochrane Database Syst Rev 2014; 4.CD010366. DOI: 10.1002/14651858.CD010366.pub2.

31. Hartley L, Dyakova M, Holmes J, Clarke A, Lee MS, ERNST E et al. Yoga for the primary prevention of cardiovascular disease. Cochrane Database Syst Rev 2014; 5.CD010072. DOI: 10.1002/14651858. CD010072.pub2.

32. Hartley L, Mavrodaris A, Flowers N, Ernst E, Rees K. Transcendental meditation for the primary prevention of cardiovascular disease. Cochrane Database Syst Rev 2014; 12.CD010359. DOI: 10.1002/14651858.CD010359.pub2.

33. Widmer RJ, Coluins NM, Coluins CS, West CP, Lerman LO, LERMAN A. Digital health interventions for the prevention of cardiovascular disease: a systematic review and meta-analysis. Mayo Clin Proc 2015; 90: 469-480. DOI: 10.1016/j.mayocp.2014.12.026.

34. Krogsbøll LT, Jørgensen KJ, GrønhøJ LarSEN C, GøTzsche PC. General health checks in adults for reducing morbidity and mortality from disease. Cochrane Database Syst Rev 2012; 10.CD009009. DOI: 10.1002/14651858.CD009009.pub2.

35. NQA.ORG. What is Qigong? National Qigong Association. [online] Available from: http://www.nqa. org/ [Accessed 22/09/2017].

36. Theodoratou E, Tzoulaki I, Zgaga L, IoAnnidis JPA. Vitamin D and multiple health outcomes: umbrella review of systematic reviews and meta-analyses of observational studies and randomised trials. BMJ. 2014; 1: g2035. DOI: https://doi.org/10.1136/ bmj.g2035.

37. Seung-Kwon M, Woong J, Belong C, Seung-Won O, SANG Min P, Bon-Kwon K et al. Efficacy of vitamin and antioxidant supplements in prevention of cardiovascular disease: systematic review and meta-analysis of randomised controlled trials. BMJ 2013; 346: f10. DOI: https://doi.org/10.1136/ bmj.f10.

38. Brown WV, Bays HE, La Forge R, Sikand G. JCL Roundtable. Gender differences in risk reduction with lifestyle changes. J Clin Lipidol 2015; 9: 486495.

39. Sommer I, Griebler U, Mahlknecht P, Thaler K, Bouskill K, GartLEhNER $G$ et al. Socioeconomic inequalities in non-communicable diseases and their risk factors: an overview of systematic reviews. BMC Public Health 2015; 15: 914. DOI: 10.1186/s12889-015-2227-y. 


\section{Appendix 1}

SUMMARY OF METHODOLOGICAL QUALITY OF INCLUDED STUDIES BASED ON THE ASSESSMENT OF EACH ITEM FROM THE AMSTAR CHECKLIST BY THIS REVIEW AUTHORS

\begin{tabular}{|c|c|c|c|c|c|c|c|c|c|c|c|}
\hline & \multicolumn{11}{|c|}{ The AMSTAR checklist } \\
\hline & 1 & 2 & 3 & 4 & 5 & 6 & 7 & 8 & 9 & 10 & 11 \\
\hline \multicolumn{12}{|c|}{ Studies reporting CVD events and/ or mortality outcomes } \\
\hline $\begin{array}{l}\text { Multiple risk factor } \\
\text { interventions }^{11}\end{array}$ & + & + & + & + & + & + & + & + & + & + & + \\
\hline Vitamin C supplementation ${ }^{12}$ & + & + & + & + & + & + & + & + & + & + & + \\
\hline Antioxidant supplements ${ }^{13}$ & + & + & + & + & + & + & + & + & + & + & + \\
\hline Vitamin D supplementation $^{14}$ & + & + & + & + & + & + & + & + & + & + & + \\
\hline Selenium supplementation ${ }^{15}$ & + & + & + & + & + & + & + & + & + & + & + \\
\hline $\begin{array}{l}\text { Vitamins and minerals } \\
\text { supplements }^{18}\end{array}$ & + & + & + & + & + & + & + & + & + & $\Theta$ & + \\
\hline Advice to reduce dietary salt ${ }^{19}$ & + & + & + & + & + & + & + & + & + & $\Theta$ & + \\
\hline Mediterranean dietary pattern ${ }^{21}$ & + & + & + & + & + & + & + & + & + & + & + \\
\hline Omega 3 fatty acids $^{25}$ & + & + & + & + & + & + & + & + & + & + & + \\
\hline Health promotion interventions ${ }^{27}$ & + & + & + & + & + & + & + & + & + & + & + \\
\hline Qigong $^{29}$ & + & + & + & + & + & + & + & + & + & + & + \\
\hline Digital health interventions $^{33}$ & + & + & + & + & + & + & + & + & + & + & + \\
\hline General health checks $^{34}$ & + & + & + & + & + & + & + & + & + & $\Theta$ & + \\
\hline \multicolumn{12}{|c|}{ Studies not reporting CVD events or mortality outcomes } \\
\hline Co-enzyme $10^{16}$ & + & + & + & + & + & + & + & + & NA & + & + \\
\hline Vitamin K supplementation $^{17}$ & + & + & + & + & + & + & + & + & NA & + & + \\
\hline $\begin{array}{l}\text { Increased consumption of fruit } \\
\text { and vegetables }\end{array}$ & + & + & + & + & + & + & + & + & NA & $\Theta$ & + \\
\hline Increased consumption of nuts ${ }^{22}$ & + & + & + & + & + & + & + & + & NA & + & + \\
\hline Green and black tea ${ }^{23}$ & + & + & + & + & + & + & + & + & NA & + & + \\
\hline Dietary fibre supplementation $^{24}$ & + & + & + & + & + & + & + & + & NA & + & + \\
\hline Omega 6 fatty acids ${ }^{26}$ & + & + & + & + & + & + & + & + & NA & + & + \\
\hline Exercise training $^{28}$ & + & + & + & + & + & + & + & + & NA & $\Theta$ & + \\
\hline Tai-Chi $^{30}$ & + & + & + & + & + & + & + & + & NA & + & + \\
\hline Yoga $^{31}$ & + & + & + & + & + & + & + & + & NA & + & + \\
\hline Transcendental meditation $^{32}$ & + & + & + & + & + & + & + & + & NA & + & + \\
\hline $\begin{array}{l}\text { 1: a priori design; } 2 \text { : duplicate study } \\
\text { publication as an inclusion criteria } 5 \\
\text { cluded studies; } 7 \text { : scientific quality of } \\
\text { appropriately in formulating conclusi } \\
\text { ncluded studies; } 10 \text { : publication bias } \\
\text { : no; NA: not applicable }\end{array}$ & & & & & 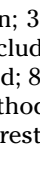 & & & 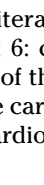 & $\begin{array}{l}\text { sea } \\
\text { acter } \\
\text { clud } \\
\text { ascu }\end{array}$ & 4: s & \\
\hline
\end{tabular}


